\title{
SSH Compromise Detection using NetFlow/IPFIX
}

Rick Hofstede, Luuk Hendriks 


\section{" 51 percent of respondents admitted that their organizations have already been impacted by an SSH key-related compromise in the last 24 months."}

-Ponemon 2014 SSH Security Vulnerability Report 


\section{SSH Compromise Detection using NetFlow/IPFIX}

Rick Hofstede, Luuk Hendriks 


\section{SSH attacks}

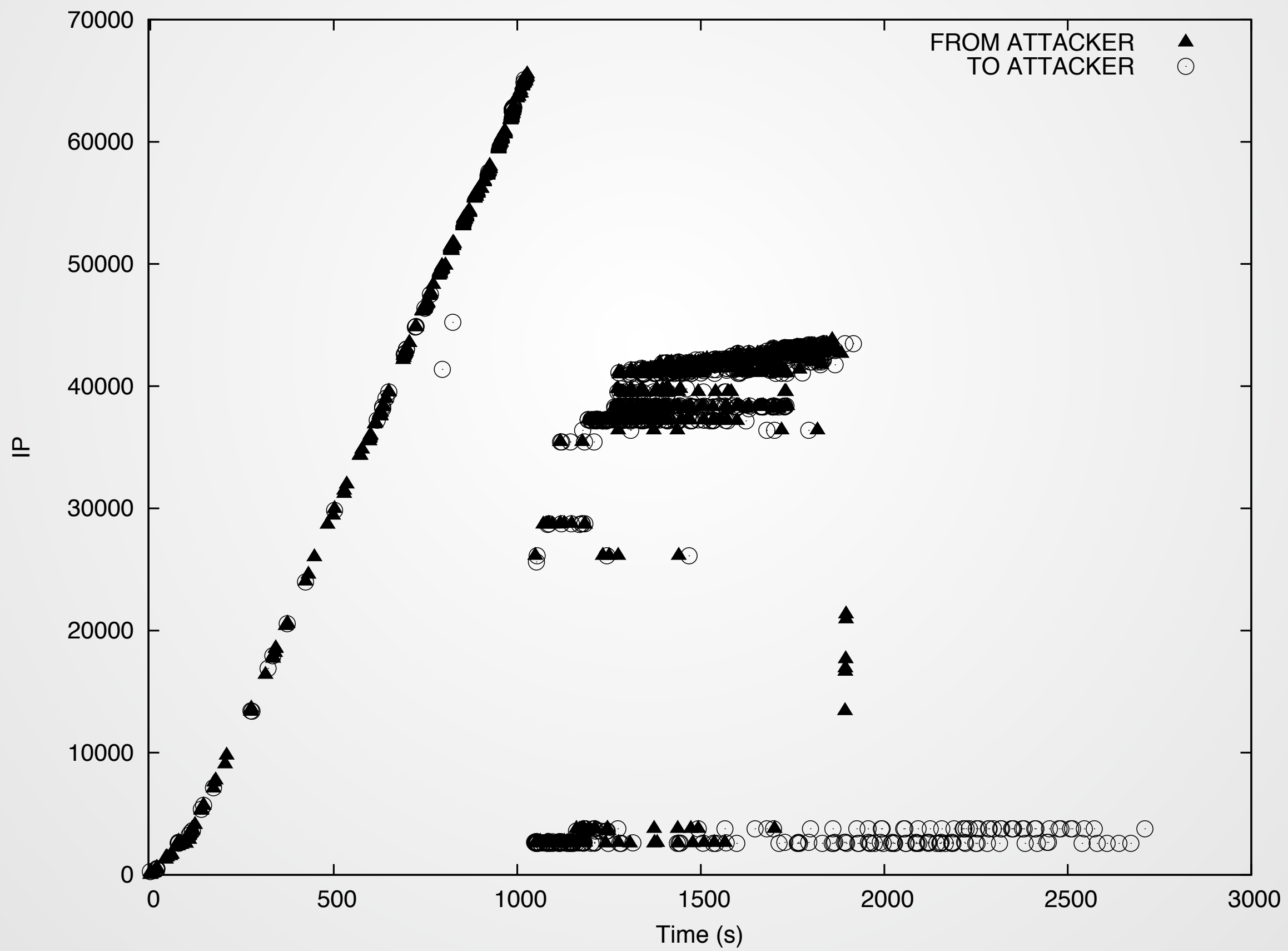




\section{SSH attacks}

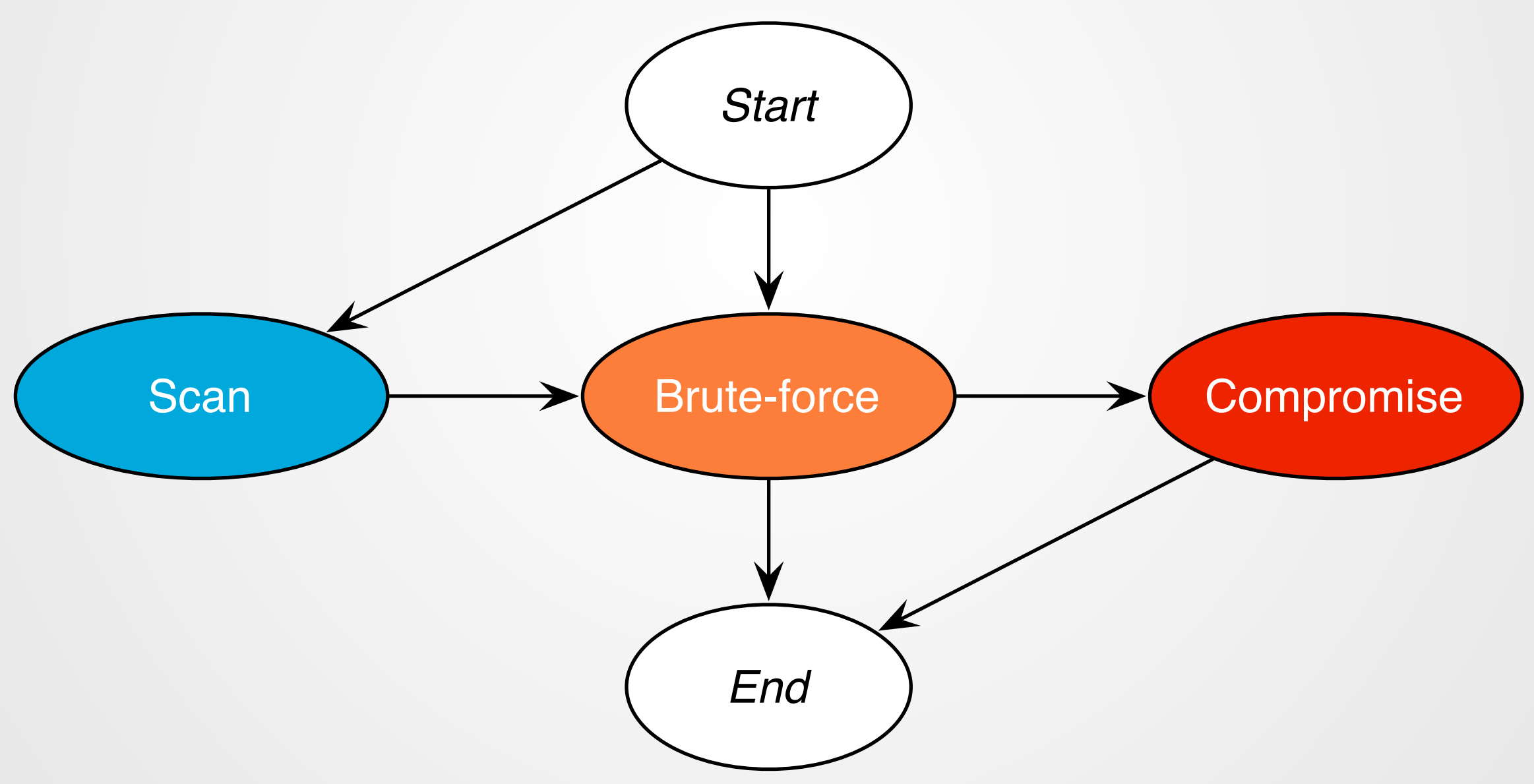




\section{SSH attacks}

- SSH intrusion detection on end hosts is hardly scalable

- Network-based approaches exist, but only inform security operators about the presence of attacks 


\section{We perform compromise detection.}




\title{
We perform compromise detection.
}

\author{
All flow-based.
}




\section{SSH attacks}

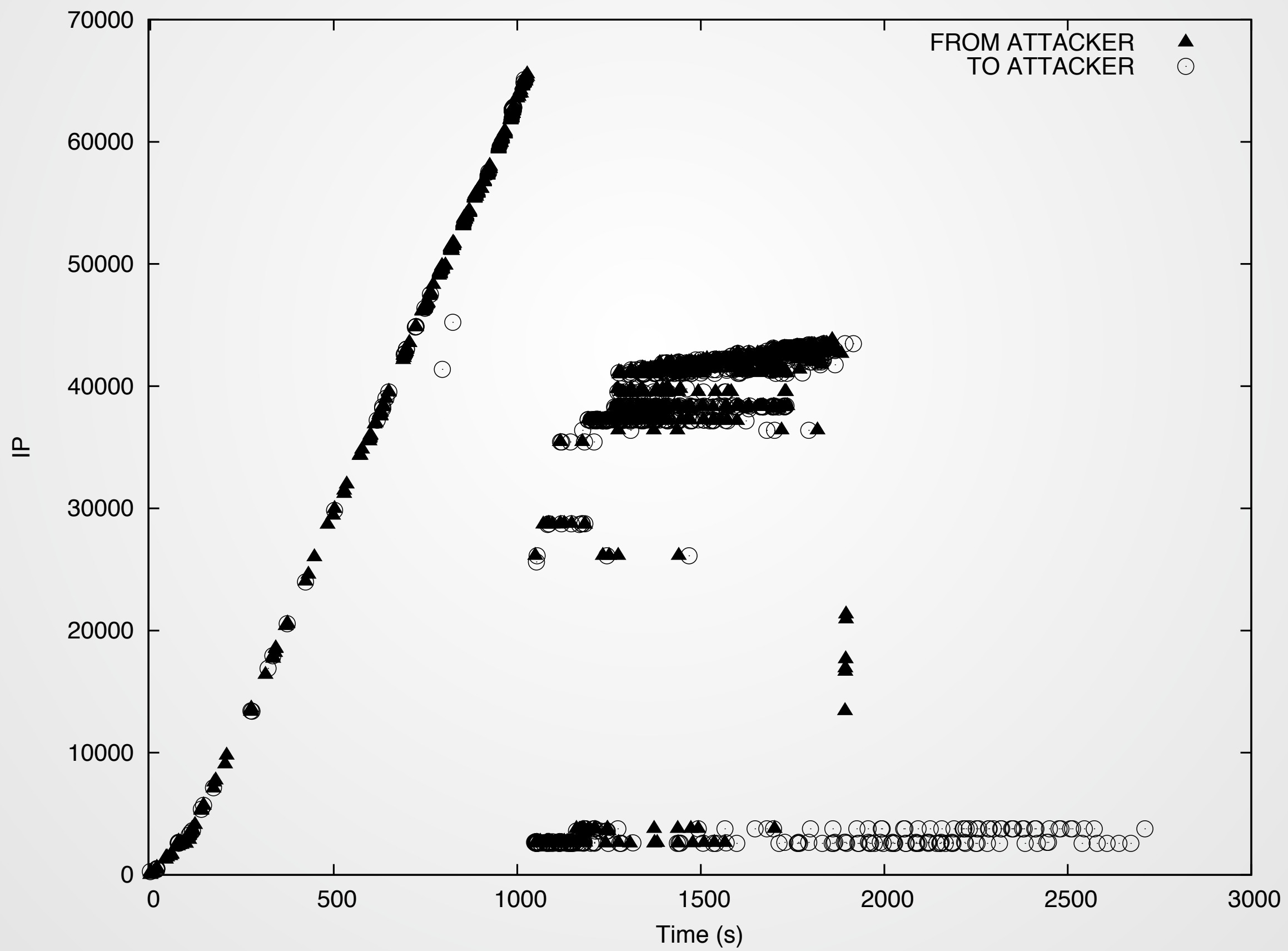




\section{SSH attacks}

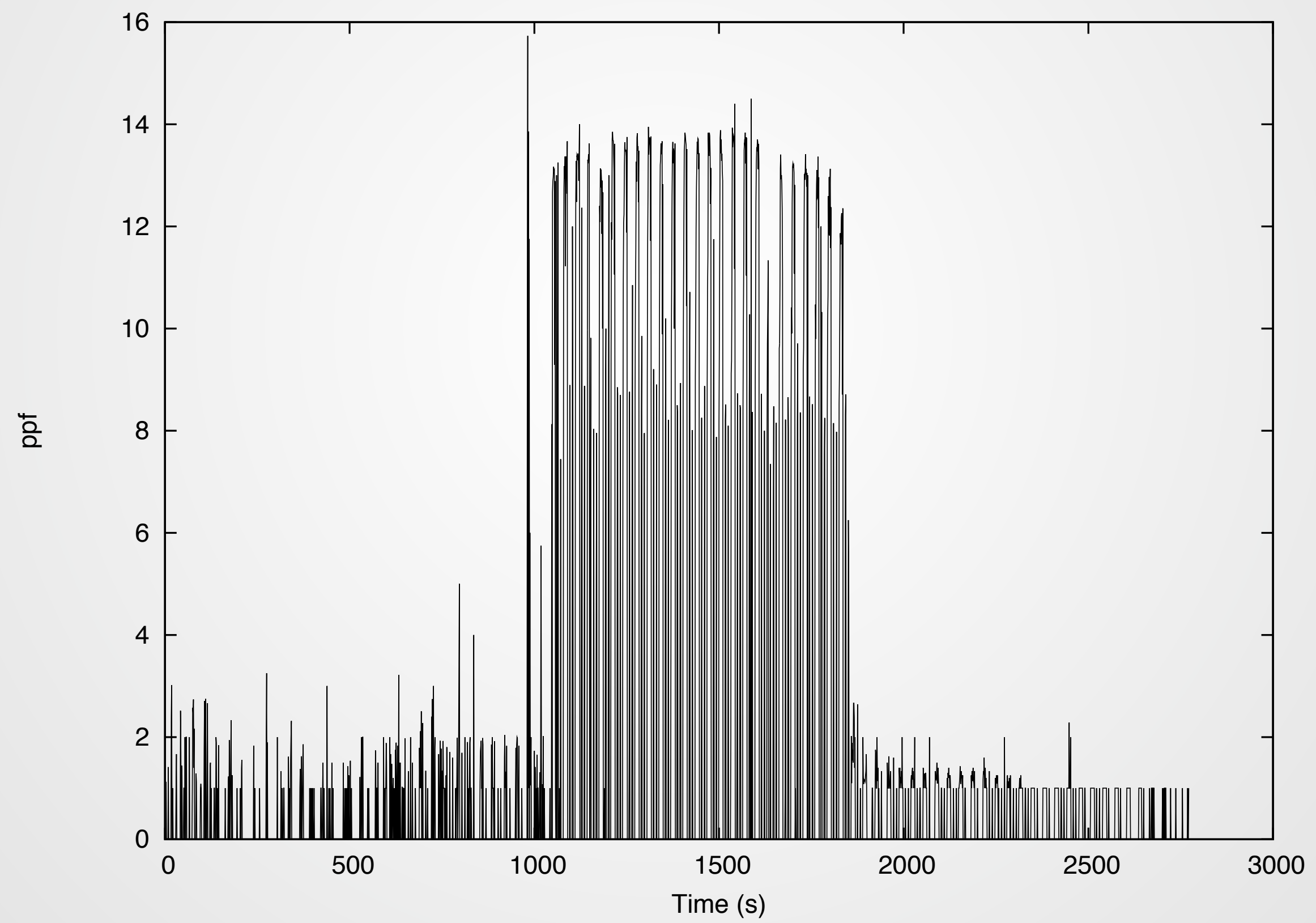




\section{A bit of history...}




\section{A bit of history...}

- SSHCure 1.0 (June '12):

- Purely deviation-based compromise detection

- SSHCure 2.0 (May '13):

- Notifications, database maintenance, performance profiling, ... 


\section{A bit of history...}

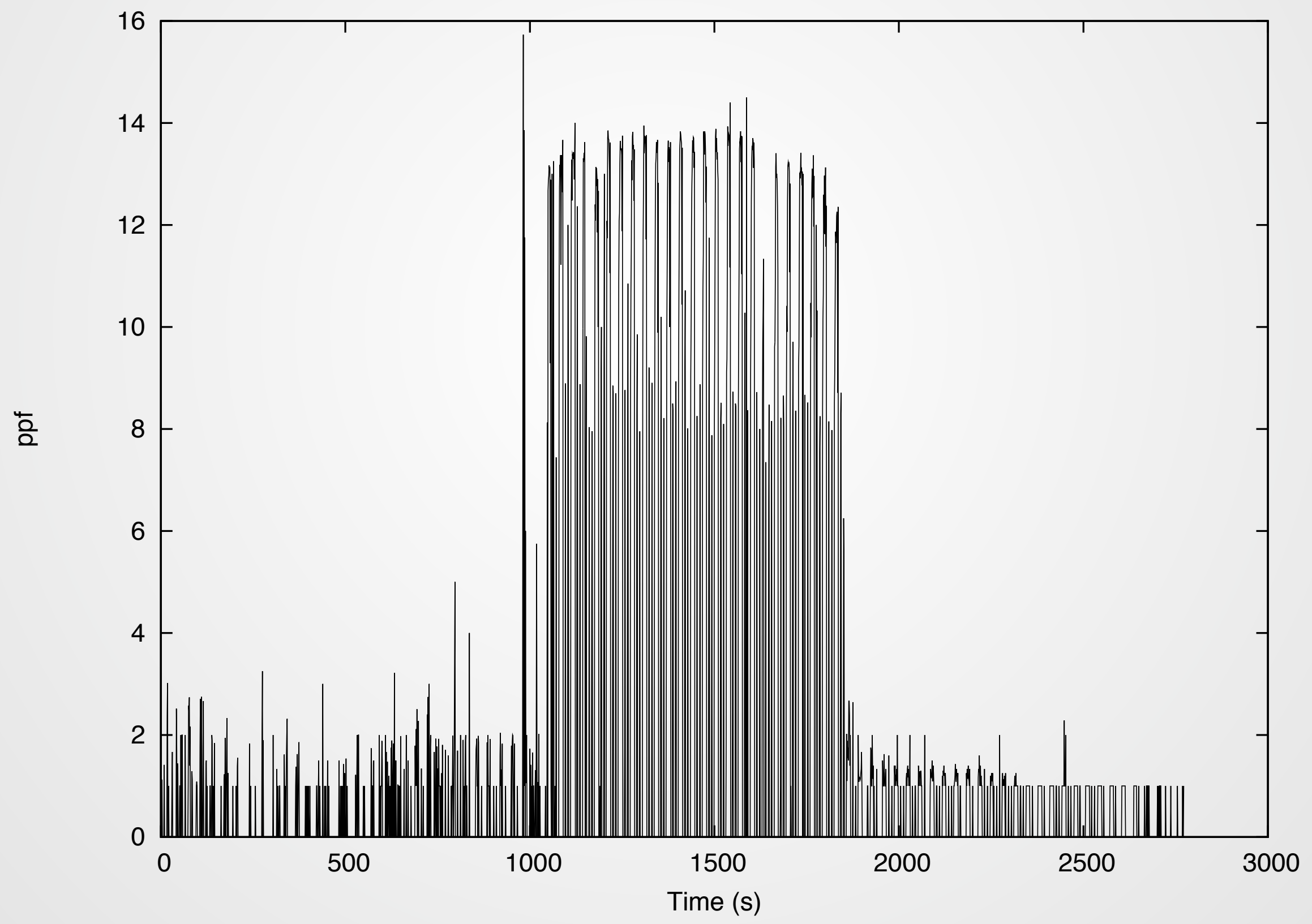




\section{A bit of history...}

- SSHCure 1.0 (June '12):

- Purely deviation-based compromise detection

- SSHCure 2.0 (May '13):

- Notifications, database maintenance, performance profiling, ... 


\section{Recent/upcoming releases}




\section{Recent/upcoming releases}

- SSHCure 2.4 (July '14):

- New compromise detection algorithm (CCR paper release), based on 'action upon compromise'

- SSHCure 3.0 (January '14):

- New frontend, ingress vs. egress attacks 


\section{Recent/upcoming releases}

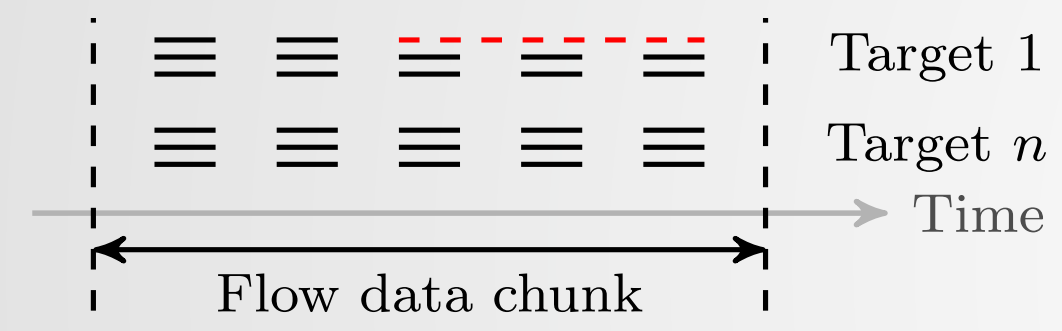

(a) Maintain connection, continue dictionary (1)

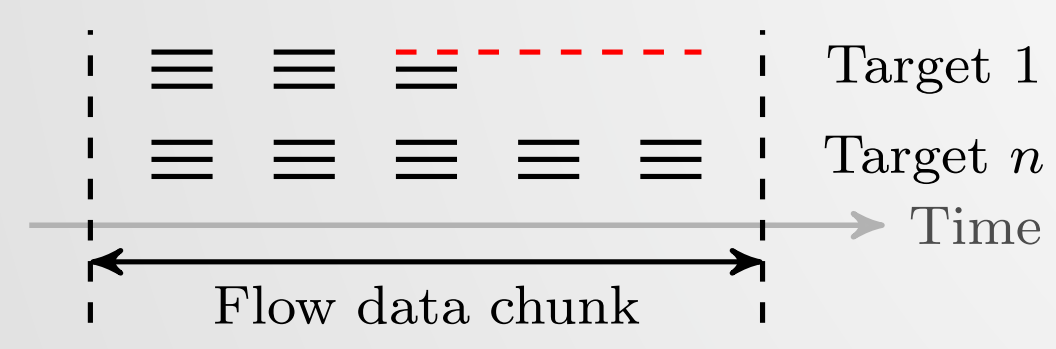

(d) Maintain connection, abort dictionary (1) 


\section{Recent/upcoming releases}

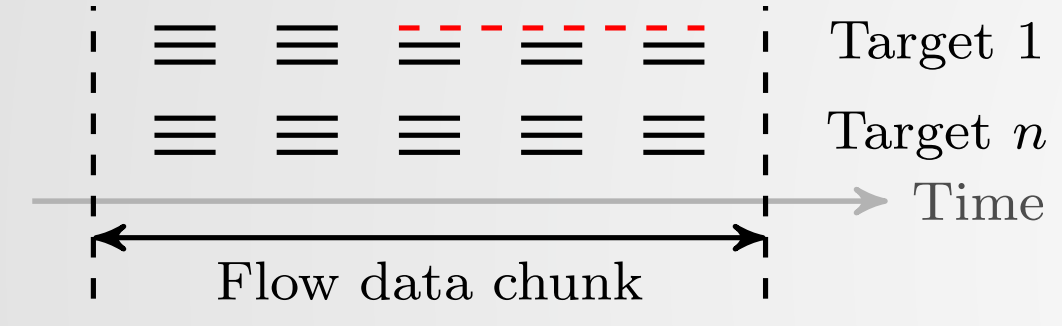

(a) Maintain connection, continue dictionary (1)

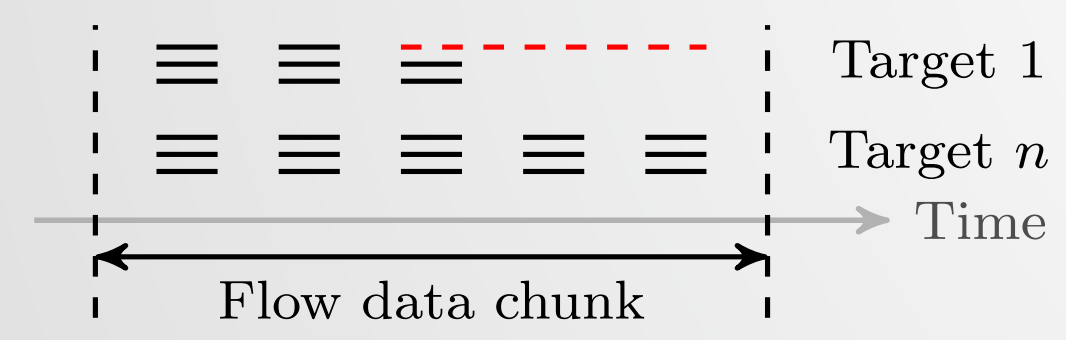

(d) Maintain connection, abort dictionary (1)

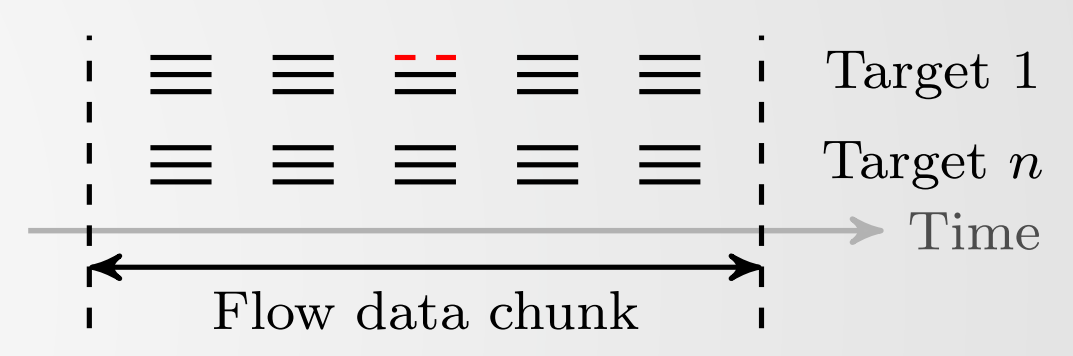

(c) Instant logout, continue dictionary

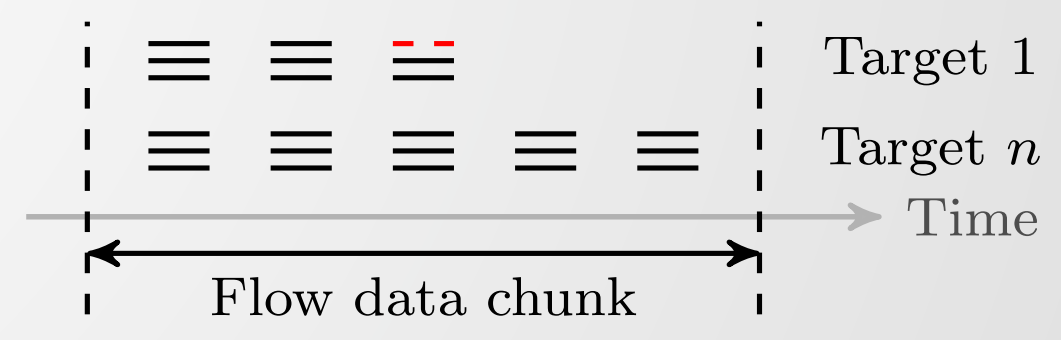

(f) Instant logout, abort dictionary 


\section{Recent/upcoming releases}

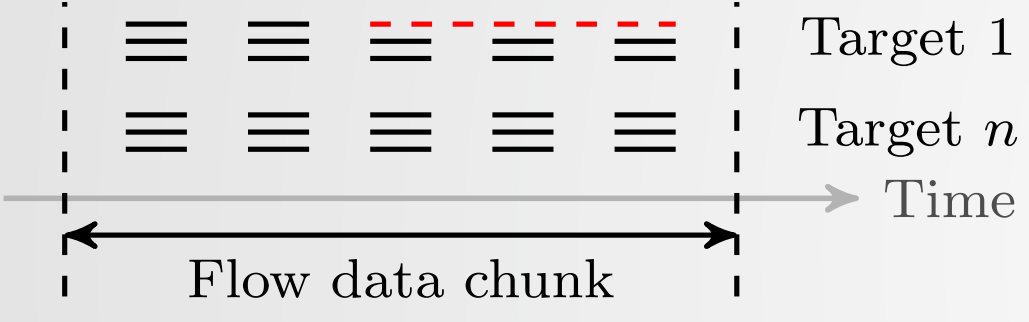

(a) Maintain connection, continue dictionary (1)

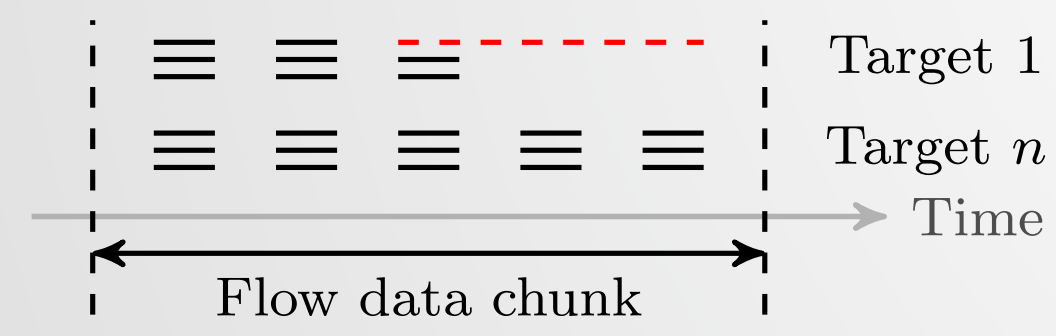

(d) Maintain connection, abort dictionary (1)

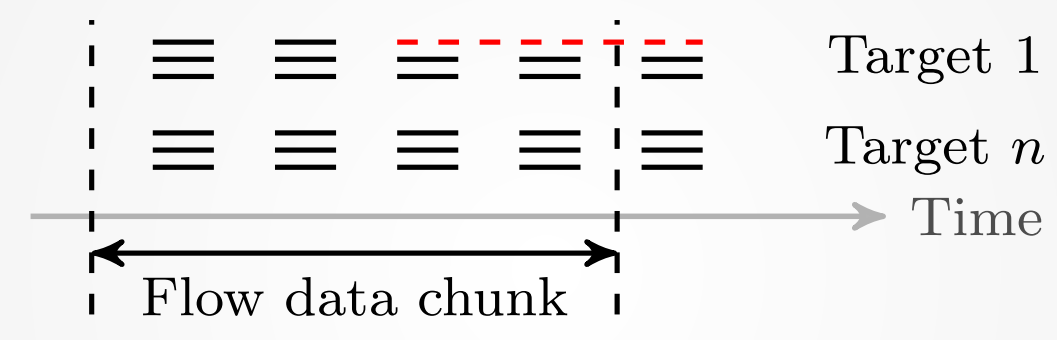

(b) Maintain connection, continue dictionary (2)

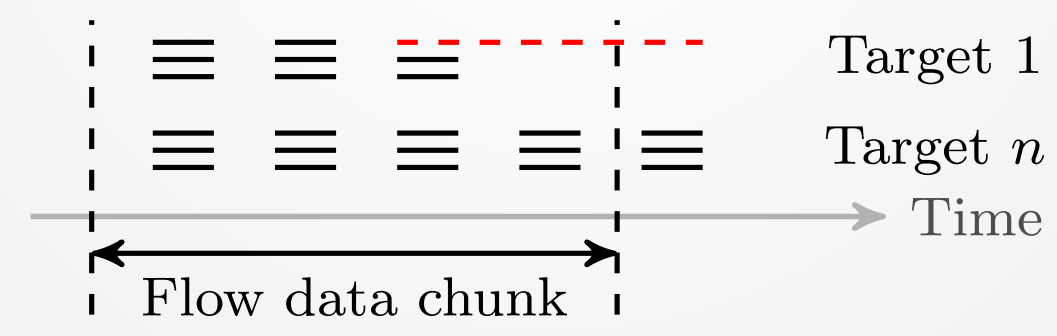

(e) Maintain connection, abort dictionary $(2)$

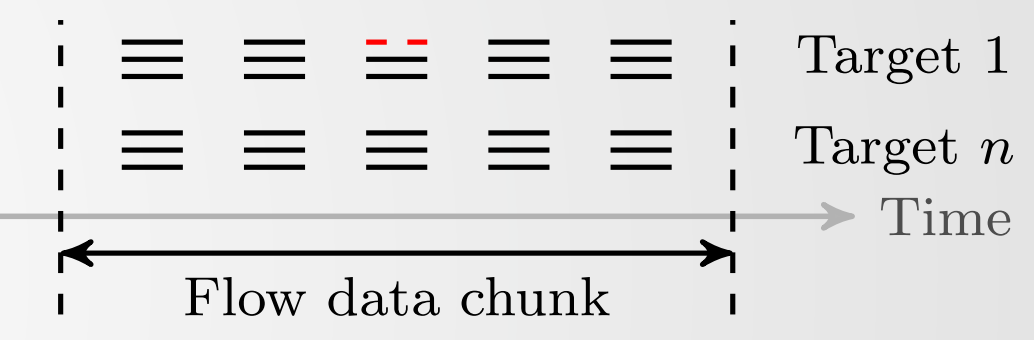

(c) Instant logout, continue dictionary

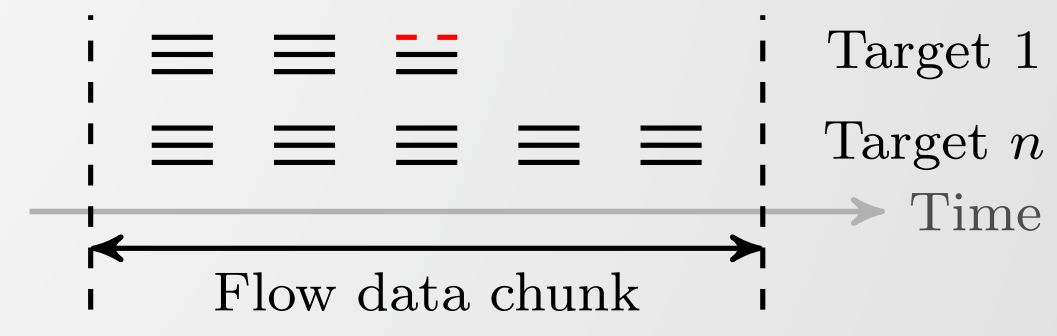

(f) Instant logout, abort dictionary 


\section{Recent/upcoming releases}

- SSHCure 2.4 (July '14):

- New compromise detection algorithm (CCR paper release), based on 'action upon compromise'

- SSHCure 3.0 (January '14):

- New frontend, ingress vs. egress attacks 


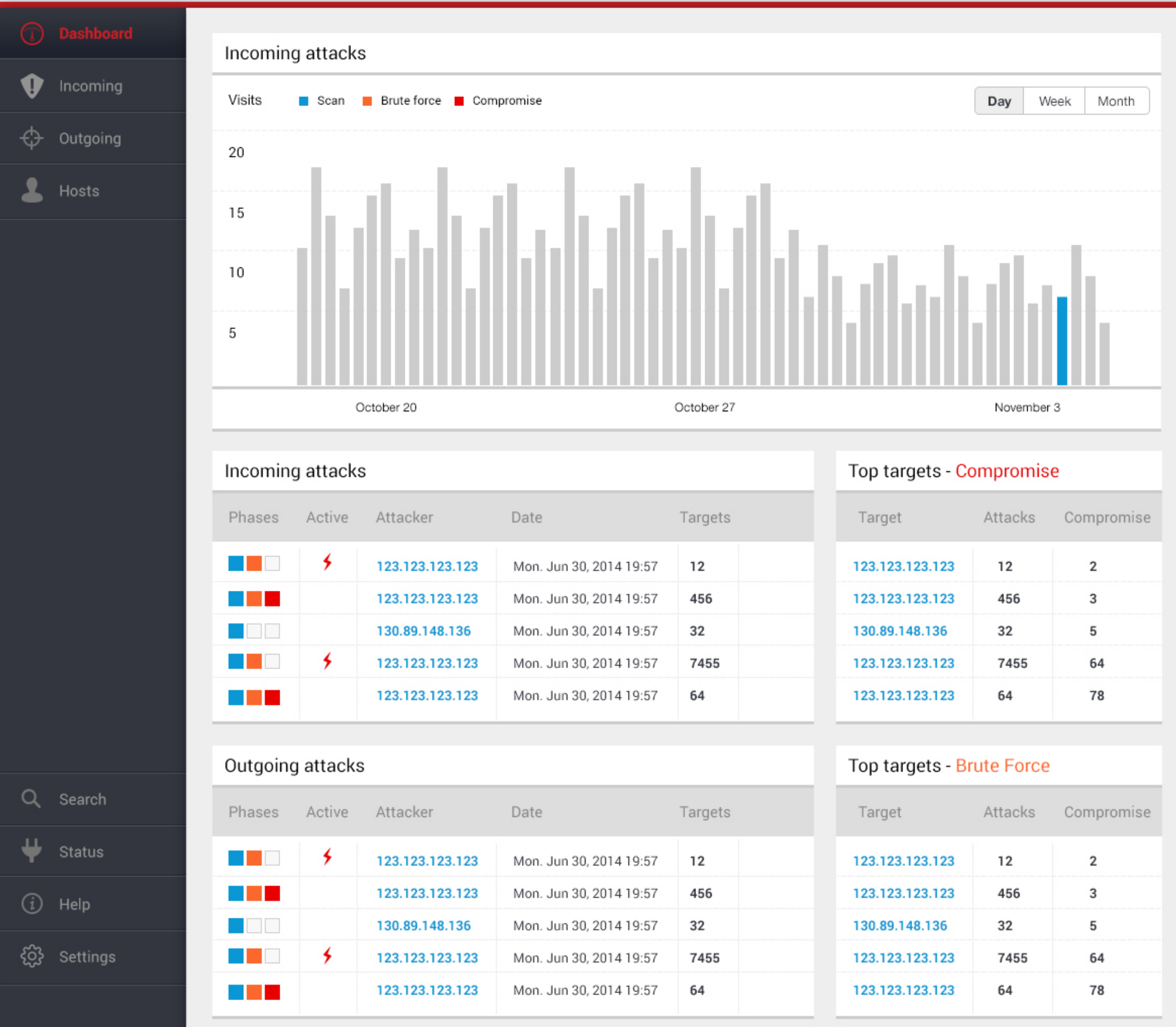




\section{SSHCure \\ Validation approach}

- Ground truth: sshd logs from 93 honeypots, servers and workstations, divided over two datasets:

- Dataset 1 - easy targets

- Dataset 2 - more difficult targets

\begin{tabular}{|l|c|c|c:c|}
\hline & Honeypots & Servers & Workstations & Attacks \\
\hline Dataset 1 & 13 & 0 & 0 & 636 \\
\hline Dataset 2 & 0 & 76 & 4 & 10353 \\
\hline
\end{tabular}




\section{SSHCure Validation results}

- Evaluation metrics:

- TP / FP — correct / false identification of incident

- TN / FN - correct / false identification of non-incident

- Detection accuracy close to $100 \%$

\begin{tabular}{|l|c|c|c|c:c|}
\hline & TPR & TNR & FPR & FNR & Acc \\
\hline Dataset 1 & 0,692 & 0,921 & 0,079 & 0,308 & 0,839 \\
\hline Dataset 2 & - & 0,997 & 0,003 & - & 0,997 \\
\hline
\end{tabular}




\section{SSHCure Deployment}

- SSHCure is open-source and actively developed

- Download counter SourceForge (Dec. '14): 3k

- Recently moved to GitHub (summer '14)

- Tested in several nation-wide backbone networks

- Many successful deployments already:

- Web hosting companies

- Campus networks
- National Research and Education Networks (NRENs)

- Governmental CSIRTs/CERTs 


\section{Lessons learned}




\section{Lessons learned}




\section{Lessons learned}

- Ease-of-use is key 


\section{Lessons learned}

- Ease-of-use is key

- Many potential SSHCure users (e.g., CSIRTs) are lessskilled than we are 


\section{Lessons learned}

- Ease-of-use is key

- Many potential SSHCure users (e.g., CSIRTs) are lessskilled than we are

- Installation scripts are important 


\section{Lessons learned}

- Ease-of-use is key

- Many potential SSHCure users (e.g., CSIRTs) are lessskilled than we are

- Installation scripts are important

- Use of NfSen: 


\section{Lessons learned}

- Ease-of-use is key

- Many potential SSHCure users (e.g., CSIRTs) are lessskilled than we are

- Installation scripts are important

- Use of NfSen:

- Widely used in (European) NREN community 


\section{Lessons learned}

- Ease-of-use is key

- Many potential SSHCure users (e.g., CSIRTs) are lessskilled than we are

- Installation scripts are important

- Use of NfSen:

- Widely used in (European) NREN community

- Experience with SURFmap [1]

[1] http://surfmap.sf.net/ 


\section{Lessons learned}




\section{Lessons learned}

- Ingress vs. egress attacks 


\section{Lessons learned}

- Ingress vs. egress attacks

- Initial focus mainly on ingress attacks 


\section{Lessons learned}

- Ingress vs. egress attacks

- Initial focus mainly on ingress attacks

- CSIRTs are becoming more responsible towards the Internet: Keep it clean! 


\section{Lessons learned}




\section{Lessons learned}

- Integration into workflow is important 


\section{Lessons learned}

- Integration into workflow is important

- Yet another tool is hard to integrate into CSIRT workflow 


\section{Lessons learned}

- Integration into workflow is important

- Yet another tool is hard to integrate into CSIRT workflow

- Integration with existing systems is necessary: IODEF, X-ARF, QuarantaineNet, ... 


\section{Lessons learned}




\section{Lessons learned}

- Advertizing is important 


\section{Lessons learned}

- Advertizing is important

- People don't spot your cool project by themselves 


\section{Lessons learned}

- Advertizing is important

- People don't spot your cool project by themselves

- Visit meetings \& conferences (FloCon, TERENA TNC, RIPE, etc.) 


\section{Lessons learned}

- Advertizing is important

- People don't spot your cool project by themselves

- Visit meetings \& conferences (FloCon, TERENA TNC, RIPE, etc.)

- GitHub vs. SourceForge 


\section{Lessons learned}




\section{Lessons learned}

- 1:1 sampling is hardly used by non-academia 


\section{Lessons learned}

- 1:1 sampling is hardly used by non-academia

- Problem for our algorithms 


\section{Lessons learned}

- $1: 1$ sampling is hardly used by non-academia

- Problem for our algorithms

- Admins are 'afraid' of increasing sampling rates 


\section{Lessons learned}




\section{Lessons learned}

- Input data quality is hard to predict 


\section{Lessons learned}

- Input data quality is hard to predict

- Algorithms should be as resilient to various data sources as possible 


\section{Lessons learned}

- Input data quality is hard to predict

- Algorithms should be as resilient to various data sources as possible

- Examples: 


\section{Lessons learned}

- Input data quality is hard to predict

- Algorithms should be as resilient to various data sources as possible

- Examples:

- Availability of TCP flags 


\section{Lessons learned}

- Input data quality is hard to predict

- Algorithms should be as resilient to various data sources as possible

- Examples:

- Availability of TCP flags

- Assumptions on flow cache entry expiration 


\section{Thanks!}

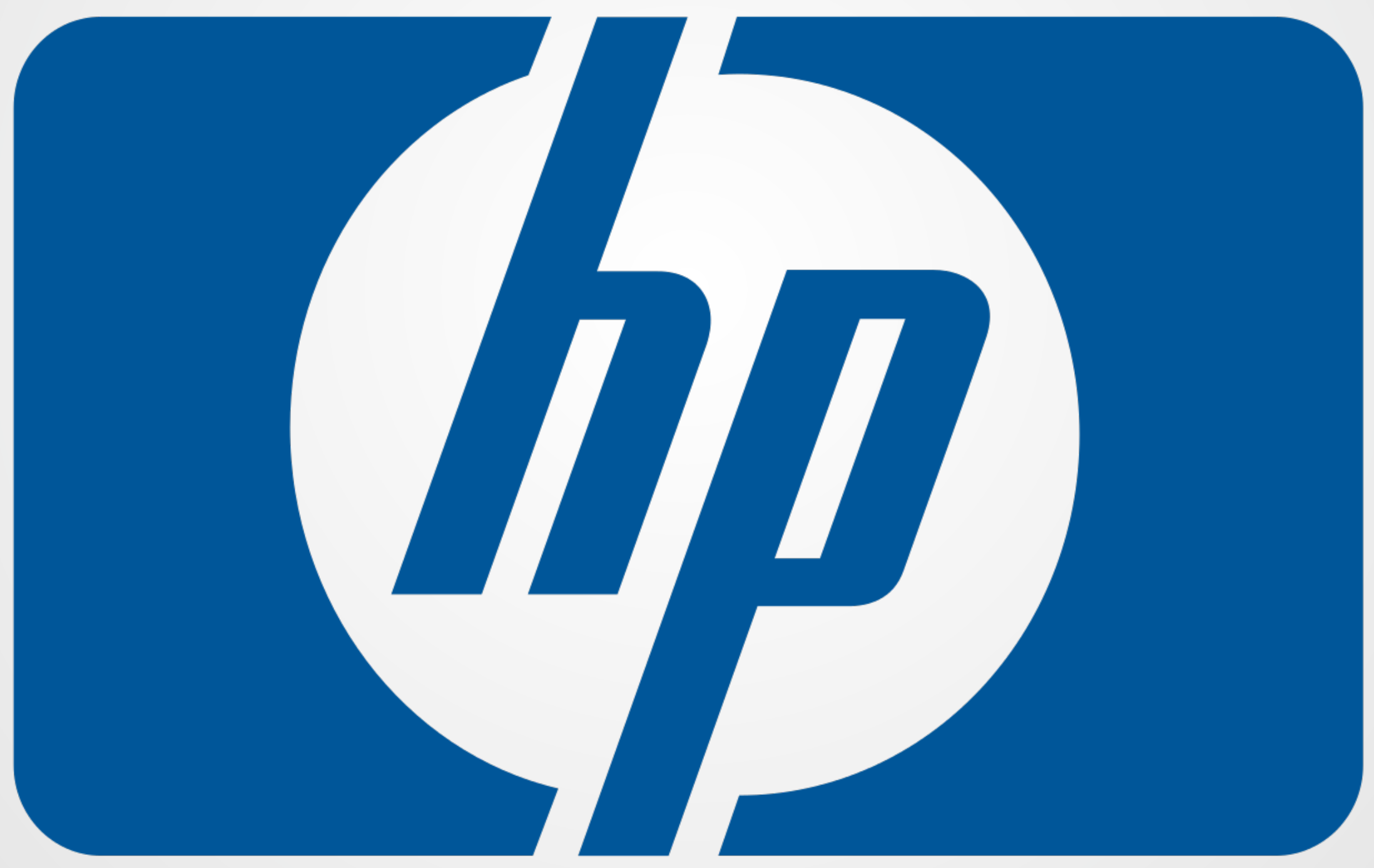




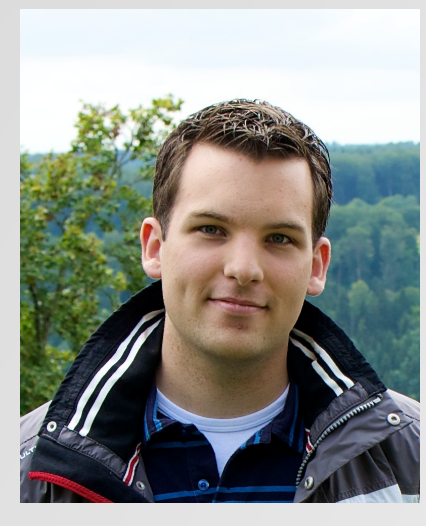

\author{
in https://nl.linkedin.com/in/rhofstede/ \\ www http://rickhofstede.nl \\ @ r.j.hofstede@utwente.nl, \\ rick.hofstede@redsocks.nl \\ in http://nl.linkedin.com/in/luukhendriks \\ www https://luukhendriks.eu \\ @ luuk.hendriks@utwente.nl
}

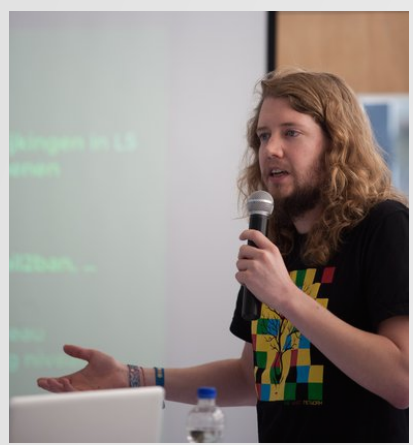

\title{
Questions?
}

\author{
https://github.com/sshcure/sshcure
}

